\title{
Scale for Measuring Perceived Service Quality of Public Service in Sri Lanka: With Special Reference to Divisional Secretariats in Gampaha District
}

\author{
A.T. Wijesekera ${ }^{1} \&$ R. Lalitha S. Fernando ${ }^{2}$ \\ ${ }^{1}$ University of Sri Jayewardenepura, Sri Lanka \\ ${ }^{2}$ Department of Public Administration, Faculty of Management Studies and Commerce, University of Sri \\ Jayewardenepura, Sri Lanka \\ Correspondence: A.T. Wijesekera, University of Sri Jayewardenepura, Sri Lanka. \\ E-mail: achinitharanga123@gmail.com
}

Received: April 7, 2017 Accepted: April 19, 2017 Online Published: October 30, 2017

doi:10.5539/par.v6n2p1 URL: http://dx.doi.org/10.5539/par.v6n2p1

\begin{abstract}
The most accepted SERVQUAL is heavily applied to measure the service quality of Business to Customer (B2C) profit oriented organizations than for non-profit organizations. As such, this paper describes the development of a 19-item instrument for assessing customer perceptions of service quality in public service with special reference to Divisional Secretariats in Sri Lanka. To do so, both qualitative and quantitative methods were utilized in three fundamental stages recommended by Churchill (1979) and Parasuraman et.al, (1988). In following their footsteps, initially a qualitative research was undertaken in five Divisional Secretariats within Gampaha District through interviews with 50 customers from different backgrounds and affiliations which produced 42 -items with eight factors emerged. These 42 -items were included in a questionnaire and quantitative study was undertaken with 100 respondents who were current or recent customers of Divisional Secretariats within Gampatha District. To ensure the reliability and validity of the measures of service quality construct, mainly reliability test, split-half reliability and factor analysis, were used. Finally, 42-items were deduced in to 19-items and a new scale was developed to measure the service quality of Divisional Secretariats with 5 dimensions Responsiveness, Communication, Tangible, Empathy and Assurance. Among these, responsiveness dimensions could be the least important and the empathy dimension was of most concern to customers. As a closing note, limitations and further studies were discussed.
\end{abstract}

Keywords: divisional secretariats, public service, service quality, SERVQUAL

\section{Introduction}

The main aim of public sector organizations is to serve the community. As far as Sri Lanka is concerned, the need for public sector quality and productivity has been talked about very much, not just over the past few years, but over decades. The government, is therefore, burdened with several issues such as public sector reforms, unemployment, poverty alleviation and most importantly eliminating fraud and corruption. Public officers must therefore learn to appreciate the need to provide high quality service that the citizens demand while establishing managerial autonomy. Therefore, to measure the service quality of existing public service is very important to identify the areas to be improved. Unlike goods quality, which can be measured objectively by such indicators as durability and number of defects (Crosby 1979; Garvin 1983), service quality is an abstract an delusive construct because of three features unique to services: intangibility, heterogeneity, and inseparability of production and consumption (Parasuraman,et.al, 1985).

Service quality is a measure of how well the service level delivered matches customer expectations. Delivering quality service means conforming to customer expectations on a consistent basis (Lewis and Bernard 1983).Thus the service quality is fundamental for both profit and nonprofit oriented organizations. The most accepted SERVQUAL is heavily applied to measure the service quality of Business to Customer (B2C) profit oriented organizations than for non-profit organizations. In relation to public service in Sri Lanka there are many issues that have been reported regarding the service quality not just over the past few years, but over decades. It has been observed that in literature, few studies have applied SERVQUAL to measure the level of service quality 
provided by some public institutions. But no studies are available related to Divisional Secretariats in Sri Lanka. Also, there is a need of a research to develop unique service quality measures for public service in Sri Lanka since Sri Lankan public services has given least attention for the development of unique service quality measures to measure their service quality. Thus, the purpose of this study is to develop measures to examine customer perceptions on service quality over the public sector organizations with reference to Divisional Secretariats.

Divisional Secretariats are the key public service organizations controlled by Ministry of Public Administration and Management which provide many social services such Civil Registration, Issuing of Permits/Licenses, Payment of Pensions, Samurdhi Program, Social welfare, Social Benefits and development programs. In this study, the researcher has concerned that the service beneficiaries also as customers.

Therefore, the objective of this study was two-fold; (1) to develop items for measuring service quality of public service in Sri Lanka (2) to evaluate their reliability and validity.

\section{Literature}

Service quality conceptualization and measurement has been debated in verity of ways through past several years. Hence, service quality for a service organization may achieve its crucial goals that are finding and retaining satisfied or repetitive customers. In fact, service quality can be defined as a customer's perception of the overall superiority of an organization's excellence in providing service (Zeithaml, 1998).

Parasuraman et al. $(1985 ; 1988)$ suggested that the customers' appraisal of the overall service quality depends on the gap between the actual performance and their expectations and they found that customers evaluate the service quality by using five criteria like tangible, reliability, responsiveness, assurance, and empathy. Among these, the tangible dimension could be the least important and the reliability dimension was the most concerned to customers. Finally, these authors developed an instrument called SERVQUAL to measure the service quality. This has been the most widely used tool in measuring customer's perception of service quality in B2C organizations. Several researchers conducted the SERVQUAL model in different sectors in different countries that some researches confirmed the SERVQUAL model (e.g. Gabbie \& Neill, 1996; Bojanic \& Rosen, 1994; Mehta \& Durvasula, 1998; Lam \& Zhang, 1998) but some others failed (e.g. Carman, 1990; Babakus \& Boller, 1992; Brown, Churchill \& Peter, 1993; Ryan \& Cliff, 1996). In deliberation of other significant studies in the literature, it appears that service quality concept includes such as technical and functional quality (Gronroos, 1984); service product, service environment, and service delivery (Rust \& Oliver, 1994); and interaction quality, physical environment quality, and outcome quality (Brady \& Cronin, 2001).

Even though, there have been numerous criticisms towards SERVQUAL model from a lot of studies in the marketing literature, there have been several studies that examined and practiced the model as a framework in measuring service quality. These criticisms have mainly revolved around the interpretation and implementation of the instrument in the service industry (Newman, 2001; Arasli et al., 2005). One of the biggest problems in the usage of SERVQUAL measurement is its dimensional structure that the researchers in different contexts reported different factors for expectations, perceptions and gap scores, thus, shortcomings concerning its universality and divergent and convergent validity issues were have also been questioned (Buttle, 1996; Carmen, 1990; Cronin \& Taylor, 1994). Despite the criticism, SERVQUAL has been widely used since it “...provides the basic skeleton... which can be adapted or supplemented to fit the characteristics or specific research needs of a particular organization..."(Parasuraman et al., 1988 ,p. 31). "While there are some practitioners, scholars and academics who believe that this topic seems to come to the end of its life in the literature in the 2000's, still there are some opponent researchers who thinks that some industries did not hear the siren call of this concept and more adaptations and theoretical applications are required in their field" (Erdogan \& Bavik, 2008). For example, Khan (2003) suggested ECOSERV for measuring quality expectations in ecotourism.

Although, several scale have been replicated, adapted and developed to measure the service quality of different services such as SERVQUAL (Parasuraman et al., 1985; 1988). SERVPERF (Cronin \& Taylor, 1992; 1994)for hotels, clubs and travel agencies, LODGSERV (Knutson, et al., 1990) for hotels, DINESERV (Stevens, Knutson \& Patton, 1995) for food and beverage establishments, SITEQUAL (Yoo \& Donthu, 2001) for internet shopping, SERVPERVAL (Petrick, 2002) for airlines, SYSTRA-SQ (Aldlaigan \& Buttle, 2002) for bank services, E-S-QUAL (Parasuraman, Zeithaml \&Malhotra, 2005) for electronic services, SELEB (Toncar et al., 2006) for education services, RENTQUAL (Erdogan \& Bavik, 2008) for car rental services and a scale not named (Law \& Hsu, 2006) for hotel web sites. However, less attention has been paid to the development of measures of service quality in public services.

Therefore, this study aims to fill this gap in the relevant literature. Ozer (1999) (cited by Erdogan \& Bavik, 2008) suggested the development of industry specific quality measurements for a better fit to the nature of the industry. 
Nor et al. (2010) (cited by Hadiyati1, 2014) states that public sector organizations which provide customer service is one of the important factors that gives significant contribution to build good reputation and credibility in the community. Public complaints of long queues, poor service and poor physical facilities are not adequate to affect the image and the quality level of service in the public sector. In echoing to this, the current study attempts to develop new measures for assessing the perceived service quality in public services. To do so, eight steps approach proposed by Churchill (1979) and modified and used by Parasuraman, et al. (1988) will be followed. These eight steps are in turn: "specify domain of construct, generate sample of items, collect data, purify the measure, assess reliability with new data, assess construct validity and finally develop norms" (Churchill, 1979, p. 66). To operationalize these steps, grounded approach (Tabachnick \& Fidell, 1996) will be employed by the use of both quantitative- in form of interview and qualitative-in form of close ended questionnaire techniques. Churchill \& Peter (1980,p. 538) concluded that"...although measures in social sciences are never universally valid for all applications and in fact, the development of valid measures is a never-ending process, better measurement can only increase the quality of marketing research and theory...".

\section{Methodology}

Churchill (1979) stressed the necessity of constructing a sound conceptual specification while developing a new measurement. In this sense, fifty interviews were conducted on Public Days (every week Wednesdays) in May and June 2016 with customers within the five Divisional Secretariats in Gampaha District where a judgmental sampling approach was used. Interviewees asked open-ended questions about their expectations, criteria and past experiences about services. Moreover, additional ad-hoc questions were asked to clarify the given responses and enhance the productivity of the interview process. Interviewees were selected from five Divisional Secretariats within the Gampaha District. Each interview last between 5-10 minutes and tape recorded. No incentive given to respondents.

Recorded interviews were studied by following the guidelines of a content analysis to create compositions of all answers. Subsequently statements related to the respondents' quality expectations from services were carefully highlighted. Researchers generated 42 distinctive statements using SERVQUAL model for the content categorization. In order to form the factors, statements with similar characteristics were grouped. The grouping process was carried out individually and collectively and resulted with the identification of eight factors. They were Access, Certainty, Communication, Coordination, Courtesy, Reliability, Responsiveness and Tangible. Then a quantitative study was undertaken to develop a unique service quality measurement for Divisional Secretariats in Sri Lanka.

\section{Analysis and Results}

Resulting 42-items transformed in to pilot questionnaire and used to collect data for first stage validation. This stage was mainly serving the confirmation purpose of newly developed scales' psychometric properties (Chu \& Murrmann, 2006). A seven-point Likert scale (Likert, 1932) ranging from (1) 'strongly disagree' to (7) 'strongly agree' was used. The sample of the pilot study consisted of 100 respondents from Gampaha District. The questionnaire was translated in to Sinhala and both Sinhala \& English questionnaires distributed accordingly as required by the respondents. To qualify for the study, respondents had to have used the service from Divisional Secretariats during the past three months. Hundred questionnaires were distributed using non-probability judgmental sampling technique to respondents and they were requested to fill out the questionnaires in a self-administered manner. Ninety five questionnaires were returned and out of them only ninety two found to be useful representing a $92 \%$ response rate. More than half $(55 \%)$ of the respondents were male between the ages of 48-57(35\%). The respondents' last visit to Divisional Secretariat indicates in Figure 1.

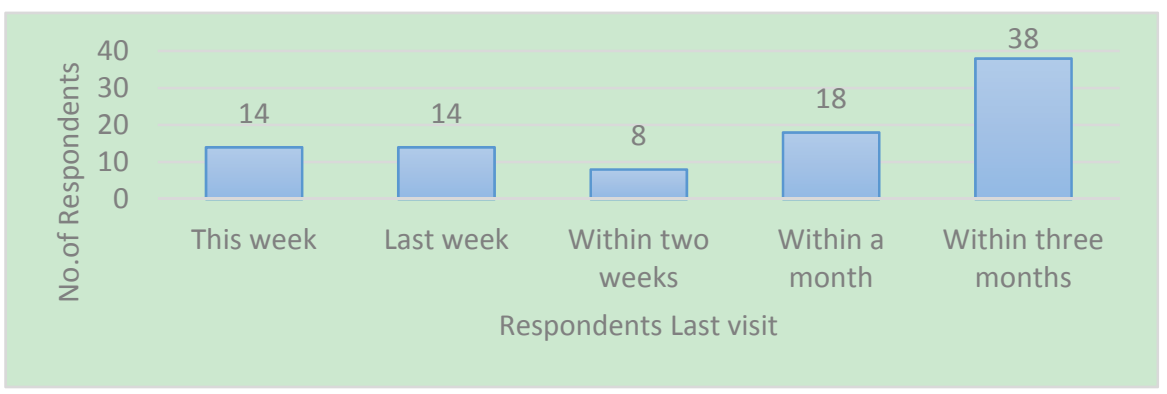

Figure 1. The respondents' last visit to Divisional Secretariat 
Churchill (1979) and Parasuraman et al. (1988) suggested that the validation of an instrument begins with the computation of Cronbach's alpha coefficient, item-to-total correlation and exploratory factor analysis (EFA). The Cronbach's Alpha value for the 42 items was .884. There was no item to be deleted. Corrected Item-Total Correlation is the correlations between each item and the total score from the questionnaire. In a reliable scale, all items should correlate with the total. So, it should be looked for items that don't correlate with the overall score from the scale: if any of these values are less than about .3 then there are a problem, because it means that a particular item does not correlate very well with the scale overall. Items with low correlations may have to be dropped. Nunnally (1970) recommended omission of the items $(<.3)$ with low corrected item-to-total correlations. The first stages of this scale development, totally 10 items were deleted from the instrument; (Table 2) and it remained only 32 -items.

Table 1. Reliability statistics

\begin{tabular}{llc}
\hline \multicolumn{2}{c}{ Cronbach's Alpha } & \multicolumn{2}{c}{ Cronbach's Alpha } \\
& Based on Standardized Items \\
\hline .87 & .884 & 42 \\
\hline
\end{tabular}


Table 2. Item-total statistics

\begin{tabular}{|c|c|c|c|c|c|}
\hline & $\begin{array}{l}\text { Scale Mean } \\
\text { if Item } \\
\text { Deleted }\end{array}$ & $\begin{array}{l}\text { Scale Variance } \\
\text { if Item } \\
\text { Deleted }\end{array}$ & $\begin{array}{l}\text { Corrected } \\
\text { Item-Total } \\
\text { Correlation }\end{array}$ & $\begin{array}{l}\text { Squared } \\
\text { Multiple } \\
\text { Correlation }\end{array}$ & $\begin{array}{l}\text { Cronbach's } \\
\text { Alpha if } \\
\text { Item Deleted }\end{array}$ \\
\hline$\overline{\mathrm{Q} 1}$ & 155.0326 & 585.373 & .373 & .732 & .873 \\
\hline Q2 & 155.8152 & 589.009 & .385 & .724 & .873 \\
\hline Q3 & 155.0326 & 589.812 & .379 & .686 & .873 \\
\hline Q4 & 156.0109 & 612.934 & .037 & .525 & .879 \\
\hline Q5 & 155.8261 & 583.398 & .493 & .645 & .871 \\
\hline Q6 & 154.8043 & 589.983 & .429 & .812 & .872 \\
\hline Q7 & 155.6739 & 612.244 & .063 & .654 & .878 \\
\hline Q8 & 155.5543 & 593.393 & .360 & .785 & .875 \\
\hline Q9 & 155.6739 & 582.464 & .478 & .814 & .871 \\
\hline Q10 & 155.6304 & 603.642 & .130 & .676 & .878 \\
\hline Q11 & 155.8261 & 609.024 & .081 & .733 & .878 \\
\hline Q12 & 155.0326 & 581.504 & .495 & .752 & .871 \\
\hline Q13 & 155.5761 & 596.686 & .350 & .755 & .875 \\
\hline Q14 & 155.7609 & 568.975 & .618 & .905 & .868 \\
\hline Q15 & 155.7391 & 565.404 & .672 & .902 & .867 \\
\hline Q16 & 155.3152 & 575.053 & .541 & .888 & .870 \\
\hline Q17 & 155.3587 & 574.320 & .609 & .893 & .869 \\
\hline Q18 & 155.2391 & 577.722 & .607 & .876 & .869 \\
\hline Q19 & 155.1196 & 581.008 & .558 & .867 & .870 \\
\hline Q20 & 155.1630 & 572.314 & .631 & .874 & .869 \\
\hline Q21 & 155.2717 & 601.101 & .387 & .798 & .875 \\
\hline Q22 & 155.5978 & 608.661 & .118 & .690 & .877 \\
\hline Q23 & 155.9891 & 613.901 & .027 & .661 & .879 \\
\hline Q24 & 156.2391 & 591.986 & .136 & .709 & .883 \\
\hline Q25 & 155.6087 & 591.603 & .391 & .869 & .873 \\
\hline Q26 & 155.3152 & 603.471 & .162 & .878 & .877 \\
\hline Q27 & 156.0435 & 584.372 & .508 & .732 & .871 \\
\hline Q28 & 155.9130 & 583.113 & .427 & .798 & .872 \\
\hline Q29 & 155.9674 & 599.241 & .301 & .693 & .877 \\
\hline Q30 & 156.2065 & 584.561 & .456 & .931 & .872 \\
\hline Q31 & 155.8696 & 590.576 & .378 & .898 & .873 \\
\hline Q32 & 155.5217 & 583.131 & .541 & .859 & .871 \\
\hline Q33 & 155.6413 & 579.419 & .609 & .843 & .870 \\
\hline Q34 & 154.9348 & 592.743 & .343 & .860 & .874 \\
\hline Q35 & 155.7609 & 579.700 & .584 & .831 & .870 \\
\hline Q36 & 155.7826 & 623.667 & $\underline{-.122}$ & .701 & .881 \\
\hline Q37 & 155.3478 & 583.526 & .517 & .819 & .871 \\
\hline Q38 & 154.9891 & 596.253 & .303 & .794 & .874 \\
\hline Q39 & 155.5978 & 580.749 & .479 & .818 & .871 \\
\hline Q40 & 155.2717 & 586.266 & .397 & .873 & .873 \\
\hline Q41 & 155.7283 & 583.870 & .508 & .822 & .871 \\
\hline Q42 & 155.4783 & 602.516 & .172 & .638 & .877 \\
\hline
\end{tabular}


Table 3. Rotated Component Matrix ${ }^{\mathrm{a}}$

\begin{tabular}{|c|c|c|c|c|c|c|c|c|}
\hline & \multicolumn{8}{|c|}{ Component } \\
\hline & 1 & 2 & 3 & 4 & 5 & 6 & 7 & 8 \\
\hline$\overline{\mathrm{Q} 1}$ & .167 & -.063 & .152 & .259 & .324 & .259 & .068 & .672 \\
\hline Q2 & .165 & .373 & -.182 & .074 & .084 &. .643 & .112 & .081 \\
\hline Q3 & -.010 & .148 & .093 & .622 & -.174 & .274 & .091 & .060 \\
\hline Q5 & .331 & .237 & .100 & .051 & -.048 & .380 & .524 & -.005 \\
\hline Q6 & .002 & .052 & .074 & .704 & .444 & .047 & .163 & .111 \\
\hline Q8 & .039 & -.106 & .154 & .164 & .029 & -.066 & .846 & .141 \\
\hline Q9 & .084 & .164 & -.033 & .390 & .361 & .142 & .576 & -.095 \\
\hline Q12 & -.146 & .224 & .329 & .392 & .140 & .326 & .499 & -.164 \\
\hline Q13 & .115 & .494 & -.050 & -.065 & -.396 & .301 & .057 & -.034 \\
\hline Q14 & .280 & .759 & .018 & .152 & -.052 & .212 & .057 & .022 \\
\hline Q15 & .275 & .784 & .117 & .197 & .032 & .137 & .081 & .075 \\
\hline Q16 & .136 & .807 & .146 & .219 & .242 & -.021 & -.163 & .094 \\
\hline Q17 & .176 & .483 & -.019 & .567 & .080 & .017 & .199 & .043 \\
\hline Q18 & .237 & .410 & .076 & .624 & .134 & -.179 & .202 & .198 \\
\hline Q19 & .052 & $\underline{.559}$ & .032 & .128 & .349 & .267 & .253 & -.031 \\
\hline Q20 & .199 & .132 & .241 & .342 & .311 & .480 & .199 & .256 \\
\hline Q21 & -.004 & .075 & .147 & .110 & .840 & .144 & .010 & -.013 \\
\hline Q25 & .499 & .300 & -.021 & .007 & -.029 &. .568 & -.318 & -.174 \\
\hline Q27 & .591 & -.106 & .224 & .159 & .010 & .385 & .084 & .040 \\
\hline Q28 & .394 & .373 & .320 & -.113 & .278 & -.203 & .308 & -.195 \\
\hline Q29 & -.011 & .288 & .072 & .160 & -.291 & -.035 & .007 &. .668 \\
\hline Q30 &.$\underline{657}$ & .201 & .204 & .283 & -.300 & .023 & -.033 & -.280 \\
\hline Q31 & .445 & .018 & .119 & .394 & -.005 & .143 & -.020 & -.485 \\
\hline Q32 & .825 & .278 & .003 & .033 & .009 & .017 & .077 & .013 \\
\hline Q33 & .738 & .363 & .153 & .032 & .145 & .062 & -.056 & .004 \\
\hline Q34 & .499 & .036 & .025 & .018 & .566 & -.190 & .193 & -.037 \\
\hline Q35 & .725 & .195 & .096 & .020 & .104 & .216 & .147 & .192 \\
\hline Q37 & .329 & -.028 & .594 & .427 & .240 & -.163 & -.034 & .149 \\
\hline Q38 & .034 & .136 & $\underline{.835}$ & -.072 & .136 & -.008 & .045 & -.134 \\
\hline Q39 & .215 & .446 & .516 & .020 & -.328 & .182 & -.022 & .211 \\
\hline Q40 & .217 & -.016 & .767 & .118 & -.014 & -.112 & .171 & .163 \\
\hline Q41 & .016 & .023 & $\underline{.680}$ & .174 & .085 & .512 & .124 & .055 \\
\hline
\end{tabular}

Extraction Method: Principal Component Analysis.

Rotation Method: Varimax with Kaiser Normalization.

a. Rotation converged in 15 iterations.

Then a Factor loadings obtained from EFA with Varimax rotation were further considered to test the factors and eliminate the poor performing items. Therefore, as the second stage of this process, Q12, Q13, Q28 \& Q31 was deleted from the instrument; (Table 3 ) and Table 4 indicates the summary of 28 items which loaded to eight factors. 
Table 4. Summary of factor lording

\begin{tabular}{|c|c|c|c|c|c|c|c|c|c|}
\hline & & Factor-1 & Factor-2 & Factor-3 & Factor-4 & Factor-5 & Factor-6 & Factor-7 & Factor-8 \\
\hline 1 & Q27 & .591 & & & & & & & \\
\hline 2 & Q30 & .657 & & & & & & & \\
\hline 3 & Q32 & .825 & & & & & & & \\
\hline 4 & Q33 & .738 & & & & & & & \\
\hline 5 & Q35 & .725 & & & & & & & \\
\hline 6 & Q14 & & .759 & & & & & & \\
\hline 7 & Q15 & & .784 & & & & & & \\
\hline 8 & Q16 & & .807 & & & & & & \\
\hline 9 & Q19 & & .559 & & & & & & \\
\hline 10 & Q37 & & & .594 & & & & & \\
\hline 11 & Q38 & & & .835 & & & & & \\
\hline 12 & Q39 & & & .516 & & & & & \\
\hline 13 & Q40 & & & .767 & & & & & \\
\hline 14 & Q41 & & & .680 & & & & & \\
\hline 15 & Q3 & & & & .622 & & & & \\
\hline 16 & Q6 & & & & .704 & & & & \\
\hline 17 & Q17 & & & & .567 & & & & \\
\hline 18 & Q18 & & & & .624 & & & & \\
\hline 19 & Q21 & & & & & .840 & & & \\
\hline 20 & Q34 & & & & & .566 & & & \\
\hline 21 & Q2 & & & & & & .643 & & \\
\hline 22 & Q20 & & & & & & .480 & & \\
\hline 23 & Q25 & & & & & & .568 & & \\
\hline 24 & Q5 & & & & & & & .524 & \\
\hline 25 & Q8 & & & & & & & .846 & \\
\hline 26 & Q9 & & & & & & & .576 & \\
\hline 27 & Q1 & & & & & & & & .672 \\
\hline 28 & Q29 & & & & & & & & .668 \\
\hline
\end{tabular}

In the third stage of this scale development process, reliability and validity were tested for new eight factors. The reliability statistics of the data set was ensured with a Cronbach's Alpha value of more than .7 (Flynn et.al 1994 cited by Chen and Paluraj, 2010) the reliability of the instrument was ensured in terms of consistency. Next step of the instrument development was to examine whether the deletion of any items could improve the Cronbach's Alpha value.

When ensuring construct validity, Exploratory Factor Analysis with Principal Component Analysis should be carried-out. To examine whether items in the scale measures the theoretical construct (Service Quality) convergent and discriminant validity have to be ensued. If an item loads significantly $<.5$ (Field, 2009, p. 648) on the factor, it is measuring the convergent validity which is prevalent and if it ensures that no other items are measured by the concept, the discriminant validity could be established.

Each factor explains a percent of the total variance. Factors that do not explain much variance Kim \& Mueller (1978) might not be worth including in the final model. It takes some iteration to come up with the optimal number of factors. Obtained the reliability and validity analysis of each factor. 


\subsection{Factor 1}

Table 5. Reliability statistics

\begin{tabular}{lcc}
\hline $\begin{array}{l}\text { Cronbach's } \\
\text { Alpha }\end{array}$ & $\begin{array}{c}\text { Cronbach's Alpha Based } \\
\text { on Standardized Items }\end{array}$ & N of Items \\
\hline .837 & .839 & 5 \\
\hline
\end{tabular}

Table 6. Item-total statistics

\begin{tabular}{llllll}
\hline & Scale Mean if & \multicolumn{2}{l}{ Scale Variance } & \multicolumn{2}{l}{ Corrected } \\
Item-Total & \multicolumn{2}{l}{ Squared MultipleCronbach's Alpha } \\
& Item Deleted & if Item Deleted & Correlation & Correlation & if Item Deleted \\
\hline Q27 & 14.2609 & 17.975 & .533 & .344 & .833 \\
Q30 & 14.4239 & 16.818 & .576 & .410 & .824 \\
Q32 & 13.7391 & 16.415 & .730 & .604 & .780 \\
Q33 & 13.8587 & 16.738 & .699 & .536 & .789 \\
Q35 & 13.9783 & 16.681 & .674 & .569 & .795 \\
\hline
\end{tabular}

The Cronbach's Alpha value for the five items included in factor 1 was .839. There was no item to be deleted and the values in the column labeled Corrected Item-Total Correlation were above .5.

Table 7. Summary -factor 1

\begin{tabular}{llll}
\hline $\begin{array}{l}\text { No of } \\
\text { Items }\end{array}$ & & $\begin{array}{l}\text { Absolute } \\
\text { loading }\end{array}$ \\
\hline $\mathbf{1}$ & Q27 & $\begin{array}{l}\text { Employee in Divisional Secretariat willing to accept complaints or criticisms } \\
\text { and at the same time }\end{array}$ & $\mathbf{. 6 8 1}$ \\
$\mathbf{2}$ & Q30 & Employee in Divisional Secretariat always available to deliver the service & $\mathbf{. 7 2 5}$ \\
$\mathbf{3}$ & Q32 & Employee in Divisional Secretariat will be efficient. & $\mathbf{. 8 5 4}$ \\
$\mathbf{4}$ & Q33 & Employee in Divisional Secretariat always ready to provide service & $\mathbf{. 8 2 7}$ \\
$\mathbf{5}$ & Q35 & $\begin{array}{l}\text { Employee in Divisional Secretariat should never be too busy to respond to } \\
\text { customer's requests }\end{array}$ & $\mathbf{. 8 1 3}$ \\
\hline
\end{tabular}

Total Variance Explained 61.24\%

All items had strong loadings on the construct, they were supposed to measure indicating uni-dimensionality and construct validity. Total Variance Explained was $61.24 \%$.

\subsection{Factor 2}

Table 8 . Reliability statistics

\begin{tabular}{ccc}
\hline Cronbach's Alpha & $\begin{array}{c}\text { Cronbach's Alpha } \\
\text { Based on Standardized } \\
\text { Items }\end{array}$ & N of Items \\
\hline .857 & .855 & 4 \\
\hline
\end{tabular}


Table 9. Item-total statistics

\begin{tabular}{llllll}
\hline & $\begin{array}{l}\text { Scale } \\
\text { if } \\
\text { Deleted }\end{array}$ & $\begin{array}{l}\text { Item } \\
\text { if Item Deleted }\end{array}$ & $\begin{array}{l}\text { Corrected } \\
\text { Item-Total } \\
\text { Correlation }\end{array}$ & $\begin{array}{l}\text { Squared } \\
\text { Multiple } \\
\text { Correlation }\end{array}$ & $\begin{array}{l}\text { Cronbach's } \\
\text { Alpha if Item } \\
\text { Deleted }\end{array}$ \\
\hline Q14 & 11.8696 & 14.071 & .720 & .608 & .810 \\
Q15 & 11.8478 & 13.427 & .800 & .685 & .774 \\
Q16 & 11.4239 & 14.159 & .724 & .539 & .808 \\
Q19 & 11.2283 & 17.167 & .571 & .348 & .867 \\
\hline
\end{tabular}

The Cronbach's Alpha value for the four items included in factor 2 was .855 . There was an item to be deleted. It was Q19. To increase the Alpha value Q19 deleted from the scale.

Table 10. Reliability statistics

\begin{tabular}{|c|c|c|}
\hline \multicolumn{3}{|c|}{ Cronbach's Alpha } \\
\hline Cronbach's Alpha & $\begin{array}{c}\text { Based on Standardized } \\
\text { Items }\end{array}$ & $\mathrm{N}$ of Items \\
\hline .867 & .867 & 3 \\
\hline
\end{tabular}

Table 11. Item-total statistics

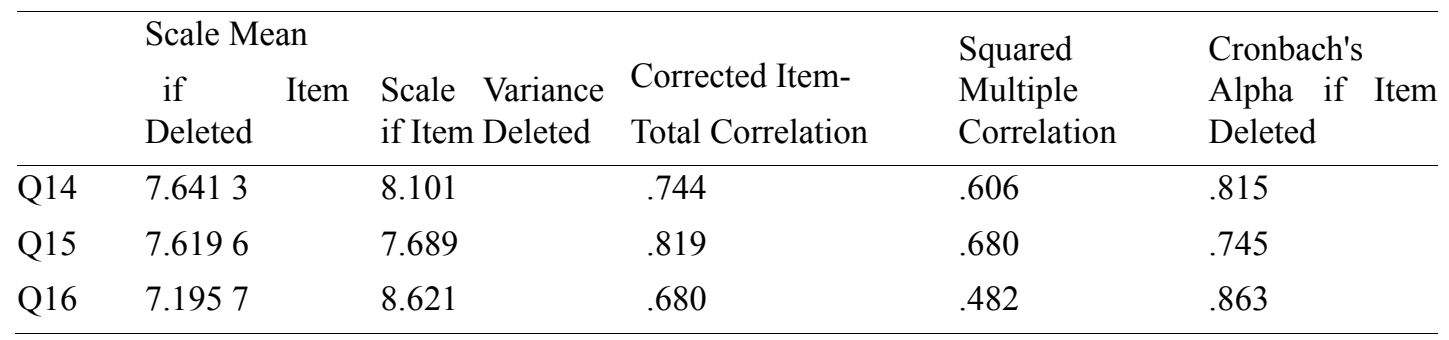

The new Cronbach's Alpha value for the five items included in factor 2 was .867 . There was no item to be deleted and the values in the column labeled Corrected Item-Total Correlation were above.3.

Table 12. Summary -factor 2

\begin{tabular}{|c|c|c|c|}
\hline $\begin{array}{l}\text { No of } \\
\text { Items }\end{array}$ & & & $\begin{array}{l}\text { Absolute } \\
\text { loading }\end{array}$ \\
\hline 1 & Q14 & $\begin{array}{l}\text { Unit functions of the Divisional Secretariat well-coordinated } \\
\text { as a team }\end{array}$ & .890 \\
\hline 2 & Q15 & Employees should have one to one effective communication & .927 \\
\hline 3 & Q16 & Employee in Divisional Secretariat will be polite and friendly & .849 \\
\hline
\end{tabular}

Total Variance Explained $79.08 \%$

All items had strong loadings on the construct, they were supposed to measure indicating uni-dimensionality and construct validity. Total Variance Explained was $79.08 \%$. 


\subsection{Factor 3}

Table 13. Reliability statistics

\begin{tabular}{ccc}
\hline Cronbach's Alpha & $\begin{array}{c}\text { Cronbach's Alpha } \\
\text { Based on } \\
\text { Standardized Items }\end{array}$ & N of Items \\
\hline .793 & .796 & 5 \\
\hline
\end{tabular}

Table 14. Item-total statistics

\begin{tabular}{lllllll}
\hline & $\begin{array}{l}\text { Scale Mean } \\
\text { if } \\
\text { Item Deleted }\end{array}$ & $\begin{array}{l}\text { Scale Variance if } \\
\text { Item Deleted }\end{array}$ & $\begin{array}{l}\text { Corrected } \\
\text { Item-Total } \\
\text { Correlation }\end{array}$ & $\begin{array}{l}\text { Squared } \\
\text { Multiple } \\
\text { Correlation }\end{array}$ & $\begin{array}{l}\text { Cronbach's } \\
\text { Alpha if } \\
\text { Deleted }\end{array}$ & \\
\hline Q37 & 15.8043 & 18.577 & .566 & .389 & .757 \\
Q38 & 15.4457 & 17.788 & .606 & .387 & .744 \\
Q39 & 16.0543 & 18.316 & .469 & .249 & .790 \\
Q40 & 15.7283 & 16.354 & .651 & .454 & .728 \\
Q41 & 16.1848 & 18.328 & .588 & .361 & .751 \\
\hline
\end{tabular}

The Cronbach's Alpha value for the five items included in factor 3 was .796. There was no item to be deleted and the values in the column labeled Corrected Item-Total Correlation were above.3.

Table 15. Summary -factor 3

\begin{tabular}{|c|c|c|c|}
\hline $\begin{array}{l}\text { No of } \\
\text { Items }\end{array}$ & & & $\begin{array}{l}\text { Absolute } \\
\text { loading }\end{array}$ \\
\hline 1 & Q37 & $\begin{array}{l}\text { Divisional Secretariat allows the implementation of service which does not } \\
\text { distinguish the class or status of the communities }\end{array}$ & .740 \\
\hline 2 & Q38 & Divisional Secretariat should be maintained in clean and tidy order & .773 \\
\hline 3 & Q39 & Divisional Secretariat have sufficient waiting area for customers & .640 \\
\hline 4 & Q40 & $\begin{array}{l}\text { Divisional Secretariat will have sufficient service providers to provide service } \\
\text { expected by customers }\end{array}$ & .806 \\
\hline 5 & Q41 & Divisional Secretariat will have Modern technology & .748 \\
\hline
\end{tabular}

Total Variance Explained 55.25\%

All items had strong loadings on the construct, they were supposed to measure the uni-dimensionality and construct validity. Total Variance Explained was 55.25\%.

\subsection{Factor 4}

Table 16. Reliability statistics

\begin{tabular}{ccc}
\hline $\begin{array}{c}\text { Cronbach's } \\
\text { Alpha }\end{array}$ & $\begin{array}{c}\text { Cronbach's Alpha Based } \\
\text { on Standardized Items }\end{array}$ & N of Items \\
\hline .746 & .748 & 4 \\
\hline
\end{tabular}


Table 17. Item-total statistics

\begin{tabular}{llllll}
\hline & $\begin{array}{l}\text { Scale Mean if } \\
\text { Item Deleted }\end{array}$ & $\begin{array}{l}\text { Scale Variance if } \\
\text { Item Deleted }\end{array}$ & $\begin{array}{l}\text { Corrected } \\
\text { Item-Total } \\
\text { Correlation }\end{array}$ & $\begin{array}{l}\text { Squared Multiple } \\
\text { Correlation }\end{array}$ & $\begin{array}{l}\text { Cronbach's Alpha } \\
\text { if Item Deleted }\end{array}$ \\
\hline Q3 & 12.6413 & 10.672 & .405 & .167 & .764 \\
Q6 & 12.4130 & 10.685 & .504 & .298 & .707 \\
Q17 & 12.9674 & 9.307 & .585 & .474 & .661 \\
Q18 & 12.8478 & 9.185 & .688 & .555 & .603 \\
\hline
\end{tabular}

The Cronbach's Alpha value for the four items included in factor 2 was .748. There was an item to be deleted. It was Q3. To increase the Alpha value, Q19 was deleted from the scale.

Table 18. Reliability statistics

\begin{tabular}{ccc}
\hline $\begin{array}{c}\text { Cronbach's } \\
\text { Alpha }\end{array}$ & $\begin{array}{c}\text { Cronbach's Alpha } \\
\text { Based on } \\
\text { Standardized Items }\end{array}$ & N of Items \\
\hline .764 & .763 & 3 \\
\hline
\end{tabular}

Table 19. Item-total statistics

\begin{tabular}{lllllc}
\hline & $\begin{array}{l}\text { Scale Mean } \\
\text { If }\end{array}$ & $\begin{array}{l}\text { Scale Variance } \\
\text { if }\end{array}$ & $\begin{array}{l}\text { Corrected } \\
\text { Item-Total } \\
\text { Correlation }\end{array}$ & $\begin{array}{l}\text { Squared } \\
\text { Multiple } \\
\text { Correlation }\end{array}$ & $\begin{array}{l}\text { Cronbach's } \\
\text { Alpha if Item } \\
\text { Deleted }\end{array}$ \\
\hline Q6 & 8.0978 & 6.199 & .472 & .269 & .709 \\
Q17 & 8.6522 & 4.933 & .599 & .464 & .682 \\
Q18 & 8.5326 & 4.779 & .735 & .552 & .520 \\
\hline
\end{tabular}

The new Cronbach's Alpha value for the three items included in factor 3 was .763. There was no item to be deleted and the values in the column labeled Corrected Item-Total Correlation were again all above. 3 .

Table 20. Summary -factor 4

\begin{tabular}{llll}
\hline $\begin{array}{l}\text { No of } \\
\text { Items }\end{array}$ & & \multicolumn{1}{c}{$\begin{array}{l}\text { Absolute } \\
\text { loading }\end{array}$} \\
\hline $\mathbf{1}$ & Q6 & $\begin{array}{l}\text { Employee in the Divisional Secretariat Should have the capability to answer } \\
\text { customer's questions }\end{array}$ & $\mathbf{. 5 3 3}$ \\
$\mathbf{2}$ & Q17 & $\begin{array}{l}\text { Employee in Divisional Secretariat give explanations and instructions to their } \\
\text { customers on a friendly way }\end{array}$ & $\mathbf{. 6 9 7}$ \\
$\mathbf{3}$ & Q18 & Employee in Divisional Secretariat will respect for customers & $\mathbf{. 8 1 8}$ \\
\hline
\end{tabular}

Total Variance Explained 68.25\%

All items had strong loadings on the construct, they were supposed to measure the uni-dimensionality and construct validity. Total Variance Explained was $68.25 \%$. 


\subsection{Factor 5}

Table 21. Reliability statistics

\begin{tabular}{ccc}
\hline $\begin{array}{c}\text { Cronbach's } \\
\text { Alpha }\end{array}$ & $\begin{array}{c}\text { Cronbach's Alpha Based } \\
\text { on Standardized Items }\end{array}$ & N of Items \\
\hline .584 & .596 & 2 \\
\hline
\end{tabular}

Table 22. Item-total statistics

\begin{tabular}{llllll}
\hline & $\begin{array}{l}\text { Scale Mean } \\
\text { if Item Deleted }\end{array}$ & $\begin{array}{l}\text { Scale Variance if } \\
\text { Item Deleted }\end{array}$ & $\begin{array}{l}\text { Corrected } \\
\text { Item-Total } \\
\text { Correlation }\end{array}$ & $\begin{array}{l}\text { Squared } \\
\text { Multiple } \\
\text { Correlation }\end{array}$ & $\begin{array}{c}\text { Cronbach's Alpha } \\
\text { if Item Deleted }\end{array}$ \\
\hline Q21 & 4.4130 & 1.871 & .424 & .180 &. \\
Q34 & 4.0761 & 1.170 & .424 & .180 &. \\
\hline
\end{tabular}

The Cronbach's Alpha value for the two items included in factor was .596. It was less than .7. Therefore, this factor was deleted from the scale.

\subsection{Factor 6}

Table 23. Reliability statistics

\begin{tabular}{ccc}
\hline $\begin{array}{c}\text { Cronbach's } \\
\text { Alpha }\end{array}$ & $\begin{array}{c}\text { Cronbach's Alpha } \\
\text { Based on } \\
\text { Standardized Items }\end{array}$ & N of Items \\
\hline .659 & .661 & 3 \\
\hline
\end{tabular}

Table 24. Item-total statistics

\begin{tabular}{lllllll}
\hline & $\begin{array}{l}\text { Scale Mean if } \\
\text { Item Deleted }\end{array}$ & $\begin{array}{l}\text { Scale Variance } \\
\text { if Item Deleted }\end{array}$ & $\begin{array}{l}\text { Corrected } \\
\text { Item-Total } \\
\text { Correlation }\end{array}$ & $\begin{array}{l}\text { Squared } \\
\text { Multiple } \\
\text { Correlation }\end{array}$ & $\begin{array}{l}\text { Cronbach's } \\
\text { Alpha if } \\
\text { Deleted }\end{array}$ & Item \\
\hline Q2 & 7.9239 & 4.862 & .519 & .280 & .492 \\
Q20 & 7.2717 & 5.343 & .414 & .173 & .640 \\
Q25 & 7.7174 & 5.590 & .482 & .250 & .550 \\
\hline
\end{tabular}

The Cronbach's Alpha value for the three items included in factor 6 was .661.It was close to .7. Also there was no item to be deleted to increase the Cronbach's Alpha value but the values in the column labeled Corrected Item-Total Correlation were above.3, which was good.

Table 25. Summary -factor 6

\begin{tabular}{llll}
\hline $\begin{array}{l}\text { No of } \\
\text { Items }\end{array}$ & & $\begin{array}{l}\text { Absolute } \\
\text { loading }\end{array}$ \\
\hline $\mathbf{1}$ & Q2 & $\begin{array}{l}\text { When customer required a service from Divisional Secretariat, it is easily } \\
\text { accessible by telephone }\end{array}$ & .813 \\
$\mathbf{2}$ & Q20 & $\begin{array}{l}\text { Employee in Divisional Secretariat will treat customers courteously on the } \\
\text { phone }\end{array}$ & .716 \\
$\mathbf{3}$ & Q25 & Divisional Secretariat maintains the trustworthiness & .785 \\
\hline
\end{tabular}

Total Variance Explained 59.68\%

All items had strong loadings on the construct, they were supposed to measure uni-dimensionality and construct 
validity. Total Variance Explained was 59.68\%.

\subsection{Factor 7}

Table 26. Reliability statistics

\begin{tabular}{ccc}
\hline $\begin{array}{c}\text { Cronbach's } \\
\text { Alpha }\end{array}$ & $\begin{array}{c}\text { Cronbach's Alpha Based } \\
\text { on Standardized Items }\end{array}$ & N of Items \\
\hline .627 & .628 & 3 \\
\hline
\end{tabular}

Table 27. Item-total statistics

\begin{tabular}{llllll}
\hline & \multirow{2}{*}{$\begin{array}{l}\text { Scale Mean if } \\
\text { Item Deleted }\end{array}$} & $\begin{array}{l}\text { Scale } \\
\text { if Item Deleted }\end{array}$ & $\begin{array}{l}\text { Variance } \\
\text { Total } \\
\text { Correlation }\end{array}$ & $\begin{array}{l}\text { Item- } \\
\text { Squared } \\
\text { Correlation }\end{array}$ & $\begin{array}{c}\text { Cronbach's Alpha if } \\
\text { Item Deleted }\end{array}$ \\
\hline Q5 & 7.4674 & 6.933 & .387 & .151 & .595 \\
Q8 & 7.1957 & 5.038 & .486 & .239 & .459 \\
Q9 & 7.3152 & 6.262 & .450 & .209 & .512 \\
\hline
\end{tabular}

The Cronbach's Alpha value for the three items included in factor 7 was .620 . There was no item to be deleted to increase the Alpha value. Therefore, the Q5, Q8 \& Q9 were deleted from the scale.

4.8 Factor 8

Table 28. Reliability statistics

\begin{tabular}{ccc}
\hline $\begin{array}{c}\text { Cronbach's } \\
\text { Alpha }\end{array}$ & $\begin{array}{c}\text { Cronbach's Alpha Based } \\
\text { on Standardized Items }\end{array}$ & N of Items \\
\hline .430 & .430 & 2 \\
\hline
\end{tabular}

Table 29. Item-total statistics

\begin{tabular}{llllll}
\hline & $\begin{array}{l}\text { Scale Mean if } \\
\text { Item Deleted }\end{array}$ & $\begin{array}{l}\text { Scale Variance } \\
\text { if Item Deleted }\end{array}$ & $\begin{array}{l}\text { Corrected } \\
\text { Item-Total } \\
\text { Correlation }\end{array}$ & $\begin{array}{l}\text { Squared } \\
\text { Multiple } \\
\text { Correlation }\end{array}$ & $\begin{array}{l}\text { Cronbach's Alpha } \\
\text { if Item Deleted }\end{array}$ \\
\hline Q1 & 3.3804 & 2.964 & .274 & .075 &. \\
Q29 & 4.3152 & 2.658 & .274 & .075 &. \\
\hline
\end{tabular}

The Cronbach's Alpha value for the two items included in factor 8 was .430.It was less than .7. Therefore, these Q1 \& Q29 were deleted from the scale. Final stage of this scale development process, new factors were named. They were;
F1- Responsiveness
F2- Communication
F3-Tangible
F4-Empathy

F6-Assurance

There were only 19 items under five dimensions for the new sale to measure the service quality of Divisional Secretariats. To ensure more reliability of these measures, Split -half reliability was concerned. This SPSS output indicates the all these data were supportive of the reliability of the measurement. 
Table 30. Reliability statistics

\begin{tabular}{llll}
\hline Cronbach's Alpha & Part 1 & Value & .850 \\
& N of Items & $10^{\mathrm{a}}$ \\
& Part 2 & Value & .827 \\
N of Items & $9^{\mathrm{b}}$ \\
& Total N of Items & 19 \\
& & .610 \\
Correlation Between Forms & .758 \\
Spearman-Brown Coefficient & Equal Length & .758 \\
Unequal Length & .752 \\
Guttman Split-Half Coefficient & \\
a. The items are: Q2, Q6, Q17, Q18, Q20, Q25, Q14, Q15, Q16, Q27. \\
b. The items are: Q30, Q32, Q33, Q35, Q37, Q38, Q39, Q40, Q41.
\end{tabular}

Again to ensure the reliability of this measure, Composite Reliability (CR) and Average Varian Extracted (AVE) were calculated using following equations. The Composite Reliability indicates the reliability and internal consistency of a latent construct. A value of $C R \geq 0.6$ (Fornell \& Larker, 1981) is required in order to achieve composite reliability for a construct. The Average Variance Extracted indicates the average percentage of variation explained by the measuring items for a latent construct. AVE $\geq 0.5$ (Fornell \& Larker, 1981)is required for every construct.

$$
\begin{gathered}
\mathbf{A V E}=\sum \mathrm{K}^{2} / \mathrm{n} \\
\mathbf{C R}=\left(\sum \mathrm{K}\right)^{2} /\left[\left(\sum \mathrm{K}\right)^{2}+\left(\sum 1-\mathrm{K}^{2}\right)\right] \\
\mathrm{K}=\text { factor loading of every item } \\
\mathrm{n}=\text { number of items in a model }
\end{gathered}
$$

Table 31. AVE \& CR values

\begin{tabular}{llllll}
\hline & F1 & F2 & F3 & F4 & F6 \\
\hline Average Variance Extracted (AVE) & .612 & .790 & .552 & .499 & .596 \\
Composite Reliability (CR) & .887 & .981 & .832 & .596 & .815 \\
\hline
\end{tabular}

All AVE and CR values included in Table 31 indicate that there is a good reliability of these measures. In order to provide support for discriminant validity, Pearson correlations among the study factors were computed. For this purpose, composite scores for each factor were calculated by averaging scores representing that dimension. Table 32 shows the significant correlations among the factors. The highest correlation occurred between F2 and F4 (0.558) and reversely, the lowest correlation was found between F6 and F3 (0.284) Bauer et.al (2006) recently assessed their newly developed scales' discriminant validity by utilizing conservative Fornell/Larcker test. Fornell \& Larcker (1981) recommended that shared variance (i.e., square of the correlation) among any two constructs should be less than the average variance extracted (AVE) of each factor (Table 33).

Table 32. Pearson correlation

**. Correlation is significant at the 0.01 level (2-tailed).

\begin{tabular}{llllll}
\hline & F1 & F2 & F3 & F4 & F6 \\
\hline F1 & $\mathbf{1}$ & & & & \\
F2 & 0.518 & $\mathbf{1}$ & & & \\
F3 & 0.431 & 0.344 & $\mathbf{1}$ & & \\
F4 & 0.348 & 0.558 & 0.323 & $\mathbf{1}$ & \\
F6 & 0.531 & 0.522 & 0.284 & 0.406 & $\mathbf{1}$ \\
\hline
\end{tabular}


Table 33. Squired multiple correlation (SMC)

\begin{tabular}{llllll}
\hline & F1 & F2 & F3 & F4 & F6 \\
\hline F1 & $\mathbf{0 . 6 1 2}$ & & & & \\
F2 & 0.268 & $\mathbf{0 . 7 9 0}$ & & & \\
F3 & 0.186 & 0.118 & $\mathbf{0 . 5 5 2}$ & & \\
F4 & 0.121 & 0.311 & 0.104 & $\mathbf{0 . 4 9 9}$ & \\
F6 & 0.282 & 0.272 & 0.081 & 0.165 & $\mathbf{0 . 5 9 6}$ \\
\hline
\end{tabular}

AVE shown as italic on diagonal

\begin{tabular}{clrrrr} 
Mean & 3.513 .74 & \multicolumn{2}{c}{3.96} & 4.21 & 3.81 \\
SD & 1.01 & 1.38 & 1.03 & 1.08 & 1.06
\end{tabular}

AVE vs. SMC significantly indicates the discriminant validity of this measurement

Finally, the developed new scale with five dimensions was mentioned in Table 34.

Table 34. New Questionnaire for measuring Service Quality of Divisional Secretariat

\begin{tabular}{lcccccc}
\multicolumn{7}{c}{ Rank } \\
\hline SD & Disagree & SD & Neutral & SA & Agree & SA \\
\hline 1 & 2 & 3 & 4 & 5 & 6 & 7
\end{tabular}

\section{F1- Responsiveness}

1 Openness - Employee in Divisional Secretariat are willing to accept complaints or criticisms and at the same time

2 Employees in Divisional Secretariat are always available to deliver the service

3 Employees in Divisional Secretariat will be efficient

4 Employees in Divisional Secretariat are always ready to provide service

5 Employees in Divisional Secretariat should never be too busy to respond to customer's requests

\section{F2-Communiocation}

6 Unit functions of the Divisional Secretariat well-coordinated as a team

7 Employees should have one to one effective communication

8 Employees in Divisional Secretariat will be polite and friendly

\section{F3- Tangible}

9 Divisional Secretariat allows the implementation of service which does not distinguish the Class or status of the communities -Fair in service

10 Divisional Secretariat should be 
maintained in a clean and tidy order

11 Divisional Secretariat provides

sufficient waiting area for customers

12 Divisional Secretariat will have sufficient service providers to provide expected service by customers

13 Divisional Secretariat will have Modern technology

\section{F4-Empathy}

14 Employees in the Divisional Secretariat Should have the capability to answer customer's questions

15 Employees in Divisional Secretariat give explanations and instructions to their customers on a friendly way

16 Employees in Divisional Secretariat will Respect for customers

\section{F5- Assurance}

17 When customer required a service from Divisional Secretariat, it is easily accessible by telephone

18 Employees in Divisional Secretariat will treat customers courteously on the phone

19 Divisional Secretariat maintains the trustworthiness

\section{Discussion and Conclusion}

This paper aims to develop a measurement scale for measuring the services quality of Divisional Secretariats as a case. To do so, scale development steps recommended by Churchill (1979) and Parasuraman et al. (1988) followed. Qualitative study was undertaken to develop 42- items emerged eight factors. They are Access, Certainty, Communication, Coordination, Courtesy, Reliability, Responsiveness and Tangible. After that, quantitative study was employed to purify the scale items, examine dimensionality, reliability, factor structure and validity and finally, 19-item scale with 5 factors Responsiveness, Communication, Tangible, Empathy, and Assurance. Among these, responsiveness dimensions could be the least important and the empathy dimension was the most concern to customers. This study contributed to the conceptual and methodological advancement of service quality and public sector literature by developing new scale to measure service quality perception of the customers of Divisional Secretariats.

Analysis of findings revealed that empathy, with the mean score of 4.21 is the most important factor in public services. Respondents stated Employee in the Divisional Secretariat should have the capability to answer customer's questions and give explanations and instructions to them on a friendly way. Also customers expect the respect from contact personnel. Second most important factor found to be the tangible, mean score is 3.96 . Respondents expect more facilities like cleanliness and order, enough waiting area, modern technology, enough service providers and fair in service. Assurance found to be the next most important factor (mean score 3.81).Customers should have the easy accessible by telephone, courtesy and trustworthiness. Communication means score is 3.74. Respondents reported that effective one to one communication between the employees and the team work is very important to provide a better service. Responsiveness has the lowest mean score it is 3.51 . Respondents reported that they expect, service providers always being available and readiness to provide the service. Also they never too busy to respond to beneficiary's requests. In this study responsiveness is the least important factor. The reason for this low ranking can be the fact that the government has allocated a public's day for every week to serve more efficient service for beneficiaries and the employees are always being 
available on public's day to deliver the service.

However, in the SERVQUAL model development process Parasuraman et.al (1988) used four clusters like Banks, Credit Card Companies, Repair - Maintenance Companies and Telephone Companies. Finally, analysis of findings revealed that reliability is consistently the most critical dimension. Assurance is the second most important dimension in all four cases. Tangible is more important in the case of the bank than in the other three firms, while the reverse was true for responsiveness. Empathy is the least important dimension in all four cases.

A comparison between new scale and SERVQUAL is given in Table 35.

Table 35. Comparison with SERVQUAL and New Scale

\begin{tabular}{ll}
\hline \multicolumn{1}{c}{ ITEMS OF NEW SCALE } & \multicolumn{1}{c}{ ITEMS OF SERVQUAL } \\
\hline \multicolumn{2}{c}{ RESPONSIVENESS } \\
$\begin{array}{l}\text { Openness - Employee are willing to accept } \\
\text { complaints or criticisms and at the same time }\end{array}$ & $\begin{array}{l}\text { It is realistic for customers to expect prompt service } \\
\text { from employees of these firms. }\end{array}$ \\
$\begin{array}{l}\text { Employees are always available to deliver the } \\
\text { service. }\end{array}$ & $\begin{array}{l}\text { They should be expected to tell customers exactly } \\
\text { when services will be performed. } \\
\text { Employee will be efficient. }\end{array}$ \\
& $\begin{array}{l}\text { Their employees always have to be willing to help } \\
\text { customers. } \\
\text { Employees are always ready to provide service. }\end{array}$ \\
$\begin{array}{l}\text { Should never be too busy to respond to customer } \\
\text { request promptly. }\end{array}$
\end{tabular}

Should never be too busy to respond to customer's requests.

\section{TANGIBLE}

Fair in service

Clean and tidy order

Sufficient waiting area for customers

Modern Technology

Sufficient service providers
The appearance of the physical facilities of these firms should be in keeping with the type of services provided.

Employees should be well dressed and appear neat

Physical facilities should be visually appealing.

Should have up-to-date equipment.

\section{EMPATHY}

Employee Should have the capability to answer customer's questions

Employee give explanations and instructions to their customers on a friendly way

Employee will respect for customers
These firms should be expected to give customers individual attention.

Employees of these firms can be expected to give customers personal attention

It is realistic to expect employees to know what the needs of their customers are.

It is realistic to expect these firms to have their customers' best interests at heart.

They should be expected to have operating hours convenient to all their customers.

\section{ASSURANCE}

When customer required a service it is easily accessible by telephone

Employee will treat customers courteously on the phone.

Maintains the trustworthiness.
Customers should be able to feel safe in their transactions with these firms' employees

Their employees should be polite.

Customers should be able to trust employees of these 
firms

Their employees should get adequate support from these firms to do their jobs well

\section{COMMUNICATION}

\author{
Unit functions are well-coordinated as a team
}

Employees should have one to one effective communication

Employee will be polite and friendly

\section{RELIABILITY}

When these firms promise to do something by a certain time, they should do so.

When customers have problems, these firms should be sympathetic and reassuring.

These firms should be dependable

They should provide their services at the time they promise to do so.

They should keep their records accurately

\section{Limitations and Future Studies}

The findings of this research should be interpreted in the light of the following limitations. There is continuing debate on using either gap scores that is perception minus expectation (Parasuraman et al., 1986; 1991) or just perceptions (Cronin \& Taylor, 1992). The first limitations with a sample distribution, having the respondents fill out two questionnaires; one before the service usage and another after was not possible due to time and follow up constraints. As Carman (1990) cogently discussed both; expectation and perception measures cannot be used simultaneously most of the time. Regarding the limitations of the study in this respect, only the perception items were conducted.

These second limitation is the use of judgmental sampling technique as one of the non- probabilistic sampling techniques. Perhaps the use of one of the probabilistic techniques would provide more confidently the chance of generalizing the results. The sample size was 100 and it was selected only from Gampaha District. Also, the original questionnaire was translated in to Sinhala and it was sometimes felt that the real meanings expected from the items should be subject to change.

As a closing note, further studies can be recommended with large sample size covering the all island using this newly developed sale to measure the service quality of Divisional Secretariats and replication studies with other public organizations would be fruitful for further generalizations of the newly developed scale.

\section{References}

Aldlaigan, A. H., \& Buttle, F. A. (2002). SYSTRA-SQ: A new measure of bank service quality. International Journal of Service Industry Management, 13(3/4), 362-381.

Arasli, H., Mehtap-Smadi,S., \& Katircioglu, S. T. (2005). Customer service quality in the Greek Cypriot banking industry. Managing Service Quality, 15(1), 41-56. R

Babakus, E., \& Boller, G. W. (1992). An empirical assessment of the SERVQUAL scale. Journal of Business Research, 24(3), 253-268. http://dx.doi.org/10.1016/0148-2963(92)90022-4

Bauer, H. H., Falk, T., \& Hammerschmidt, M. (2006). eTrans Qual: A transaction process-based approach for capturing service quality in online shopping. Journal of Business Research, 59(7), 866-875.

Bojanic, D. C., \& Rosen, L. D. (1994). Measuring service quality in restaurants: An application of the SERVQUAL instrument. Journal of Hospitality and Tourism Research, 18(1), 3-14.

Brady, K. M., \& Cronin, J. J. (2001). Some new thoughts on conceptualizing perceived service quality: A hierarchical approach. Journal of Marketing, 65(July), 34-49. Retrieved from http://www.jstor.org/stable/3203465

Brown, T. J., Churchill Jr, G. A., \& Peter, J. P. (1993). Improving the measurement of service quality. Journal of Retailing, 69(1), 127-139.

Buttle, F. (1996). SERVQUAL:review, critique, research agenda. European Journal of Marketing, 30(1), 8-32.

Carmen, J. (1990). Consumer perceptions of service quality: An assessment of the SERVQUAL dimensions. Journal of Retailing, 66(1), 33-35. 
Chu, K. H. L., \& Murrmann, S. K. (2006). Development and validation of the hospitality emotional labor scale. Tourism Management, 27(6), 1181-1191.

Churchill Jr., G. A., \& Peter, P. (1980). Measurement abstracts: purpose, policy, and procedures. Journal of Marketing Research, 17(4), 537-538.

Churchill, G. (1979). A paradigm for developing better measures of marketing constructs. Journal of Marketing Research, 16(February), 64-73.

Cronin, J. J., \& Taylor, S. A. (1992). Measuring service quality: A reexamination and extension. Journal of Marketing, 56(July), 55-68. Retrieved from http://www.jstor.org/stable/1252296

Cronin, J. J., \& Taylor, S. A. (1994). SERVPERF versus SERVQUAL: Reconciling performance-based and perceptions- minus-expectations measurement of service quality. Journal of Marketing, 58(January), $125-131$.

Crosby, P. B. (1979). Quality is free: The art of marking quality certain. New York: New American Library.

Erdogan, H. E., \& Bavik, A. (2008). Scale Development Process: Service Quality in Car Rental Services. The Electronic Journal of Business Research Methods, 6, 133-146.

Field, A. (2009). Discovering statistics using SPSS (3rd ed.). SAGE Publications Inc., p.648.

Fornell, C., \& Larcker, D. F. (1981). Evaluating structural equation models with unobservable variables and measurement error. Journal of Marketing Research, 18(1), 39-50. Retrieved from http://www.jstor.org/stable/3151312

Frochot, I. M., \& Hughes, H. (2000). HISTOQUAL: The development of a historic houses assessment scale. Tourism Management, 21(2), 157-167.

Gabbie, O., \& Neill, M. A. (1996). SERVQUAL and Northern Ireland hotel sector: a comparative analysis-part 1. Managing Service Quality, 8(5), 306-311.

Garvin, D. A. (1983, September-October). Quality on the Line, Harvard Business Review, 61(5), 65-73.

Grönross, C. (1984). A service quality mode and its marketing implications. European Journal of Marketing, 18(4), 36-44. http://dx.doi.org/10.1108/EUM0000000004784

Hadiyatil, E. (2014). Service quality and performance of public sector: study on immigration office in Indonesia. International Journal of Marketing Studies, 6(6).

Khan, M. M. (2003). ECOSERV: Eco-tourists' quality expectations. Annals of Tourism Research, 30(1), 109-125. http://dx.doi.org/10.1016/S0160-7383(02)00032-4

Kim, J. O., \& Mueller, C. W. (1978). Introduction to factor analysis: what it is and how to do it. Beverly Hills, Calif.: Sage Publications. (HA29.Q35/VOL 13).

Knutson, B., Stevens, P., Wullaert, C., \& Yokoyoma, F. (1990). LODGSERV: A service quality index for the lodging industry. Hospitality Research Journal, 14(2), 227-284.

Lam, T., \& Zang, H. Q. (1998). Service quality of travel agents: The case of travel agents in Hong Kong. Journal of Tourism Management, 20(3), 341-349.

Law, R., \& Hsu, C. H. C. (2006). Importance of hotel website dimensions and attributes: perceptions of online browsers and online purchasers. Journal of Hospitality and Tourism Research, 30(3), 295-312.

Lewis, R. C., \& Bernard, H. B. (1983). The Marketing Aspects of Service Quality. In L. Berry, G. Shostack, \& G. Upah (Eds.), Emerging Perspectives on Services Marketing (pp. 99-107). Chicago: American Marketing.

Likert, R. (1932). A technique for the measurement of attitudes. archives of Psychology (Doctoral dissertation). 140, (eds) Woodworth, R.S., New York University.

Mehta, S. C., \& Durvasula, S. (1998), Relationship between SERVQUAL dimensions and organizational performance in the case of a business-to-business service. Journal of Business and Industrial Marketing, $13(1), 40-53$.

Newman, K. (2001). Interrogating SERVQUAL: A critical assessment of service quality measurement in a high street retail bank. International Journal of Bank Marketing, 19(3), 126-139.

Nunnally. (2017). Introduction to Psychological Measurement. New York Mc Grow Hill. Retrieved from http://trove.nal.gov.au/version/25598505 
Parasuraman, A., Berry, L., \& Zeithaml, V. A. (1986). SERVQUAL: A multiple-item scale for measuring customer perceptions of service quality. Working Paper, Cambridge: Marketing Science Institute.

Parasuraman, A., Berry, L., \& Zeithaml, V. A. (1991). Refinement and reassessment of the SERVQUAL scale. Journal of Retailing, 67(4), 420-447.

Parasuraman, A., Zeithaml, V. A., \& Berry, L. (1985). A conceptual model of service quality and its implications for future research. Journal of Marketing, 49(Fall), 41-50.

Parasuraman, A., Zeithaml, V. A., \& Berry, L. (1988). A multi item scale for measuring consumer perception of service quality. Journal of Retailing, 64(Spring), 12-40.

Parasuraman, A., Zeithaml, V. A., \& Malhotra, A. (2005). E-S-QUAL: A multiple-item scale for assessing electronic service quality. Journal of Service Research, 7(3), 213-233.

Petrick, J. F. (2002). Development of a multi-dimensional scale for measuring the perceived value of a service. Journal of Leisure Research, 34(2), 119-134.

Rust, R. T., \& Oliver, R. L. (1994). Service quality insights and managerial implications from the frontier. In Rust R. T. \& Oliver R. L. (Eds). Service quality: new directions in theory and practice (pp. 1-19). Thousand Oaks, CA: Sage Publications. http://dx.doi.org/10.4135/9781452229102.n1

Ryan, C., \& Cliff, A. (1996). Users and non-users on the expectation item of the SERVQUAL scale. Annals of Tourism Research, 23(4), 931-934. Retrieved from http://www.sciencedirect.com/science/journal/01607383/23

Stevens, P., Knutson, B., \& Patton, M. (1995). DINESERV: A tool for measuring service quality in restaurants. Cornell Hotel and Restaurant Administration Quarterly, 36(2), 56-60.

Toncar, M. F., Reid, J. S., Burns, D. J., Anderson, C. E., \& Nguyen, H. P. (2006). Uniform assessment of the benefits of service learning: the development, evaluation, and implementation of the SELEB Scale. Journal of Marketing Theory and Practice, 14(3), 223-238.

Yoo, B., \& Donthu, N. (2001). Developing a scale to measure the perceived quality of internet shopping sites (SITEQUAL). Quarterly Journal of Electronic Commerce, 2(1), 31-47.

Zeithaml, V. A. (1988). Consumer perceptions of price, quality and value: A means-end synthesis of evidence. Journal of Marketing, 52(3), 2-22. Retrieved from http://www.jstor.org/stable/1251446

\section{Copyrights}

Copyright for this articleis retained by the author(s), with first publication rights granted to the journal.

This is an open-access article distributed under the terms and conditions of the CreativeCommons Attribution license (http://creativecommons.org/licenses/by/4.0/). 